\title{
Health Care for Patients with Serious Mental Illness: Family Medicine's Role
}

\author{
Nancy E. Morden, MD, MPH, Lisa A. Mistler, MD, MS, William B. Weeks, MD, MBA, \\ and Stephen J. Bartels, MD, MS
}

Numerous studies document disproportionate physical morbidity and premature death among people with serious mental illness. Although suicide remains an important cause of mortality for this population, cardiovascular disease is the leading cause of death. Cardiovascular death among those with serious mental illness is 2 to 3 times that of the general population. This vulnerability is commonly attributed to underlying mental illness and behavior. Some excess disease and deaths result from poor access to and use of quality health care. Negative cardiometabolic effects of newer psychotropic medications augment these trends by increasing rates of obesity, diabetes, and hyperlipidemia among those treated. Researchers have developed innovative care models aimed at minimizing the disparate health outcomes of patients with serious mental illness. Most strive to enhance access to primary care, but publications on this topic appear almost exclusively in the psychiatric literature. A focus on primary care for the prevention of excess cardiometabolic morbidity and mortality in this population is appropriate, but depends on primary care physicians' understanding of the problem, involvement in the solutions, and collaboration with psychiatrists. We review health outcomes of the seriously mentally ill and models designed to improve these outcomes. We propose specific strategies for Family Medicine clinicians and researchers to address this problem. (J Am Board Fam Med 2009;22:187-195.)

\section{Serious Mental Illness: A Cardiovascular Disease Risk Equivalent?}

In 2002, the National Heart Lung and Blood institute labeled diabetes a cardiovascular disease (CVD) risk equivalent, indicating patients with diabetes have a risk of CVD events equal to that of

This article was externally peer reviewed.

Submitted 19 March 2008; revised 26 June 2008; accepted 1 July 2008.

From the Department of Community and Family Medicine (NEM, WBW), the Department of Psychiatry (LAM, WBW), Dartmouth Medical School, The Dartmouth Institute for Health Policy and Clinical Practice, Lebanon, NH; the VA National Center for Patient Safety, White River Junction, VT (WBW); and the Centers for Senior Health and Aging, Lebanon, NH (SJB).

Funding: Dr. Morden was supported in part by VA Health Services Research and Development Grant REA-03-098. Dr. Lisa Mistler was supported by VA National Quality Scholars Program training grant TPQ 97-000. Dr. William Weeks was supported in part by VA Health Services Research and Development Grant REA-03-098. Dr Steve Bartels was supported in part by NIMH grant K24MH066282.

Conflict of interest: none declared.

Corresponding author: Nancy E. Morden, MD, MPH, Assistant Professor, Department of Community and Family Medicine, Dartmouth Medical School, The Dartmouth Institute for Health Policy and Clinical Practice, 35 Centerra Parkway, Suite 300, Lebanon, NH 03766 (E-mail: Nancy.e.morden@dartmouth.edu). people with known CVD. ${ }^{1}$ This information represented a paradigm shift in cardiovascular event prevention for diabetics. A similar shift may be warranted for people with serious mental illness (SMI) (schizophrenia spectrum, bipolar disorder, and refractory depression). The association between SMI and cardiovascular event risk has not been conclusively quantified, but the high prevalence of cardiometabolic risk factors, CVD, and cardiovascular death in this population may justify classification of SMI, like diabetes, as a CVD risk equivalent. At very least SMI should signal a need for attentive cardiovascular risk assessment and prevention efforts. Such a shift in understanding will focus physicians on the cardiometabolic hazards this group faces and prompt appropriate attention to the problem. In this article we aim to convince family physicians of the need for this paradigm shift and to engage them in the effort to improve health and health care for this population.

\section{Background}

Health outcomes and life expectancy of patients with SMI are poor and worsening. Much of this 
Table 1. Minimal Monitoring Protocol for People Taking Second-Generation Antipsychotics ${ }^{5}$

\begin{tabular}{|c|c|c|c|c|c|c|c|}
\hline & Baseline & 4 Weeks & 8 Weeks & 12 Weeks & Quarterly & Annually & Every 5 Years \\
\hline Personal/family history & $\mathrm{x}$ & & & & & $\mathrm{x}$ & \\
\hline Weight (BMI) & $\mathrm{x}$ & $\mathrm{x}$ & $\mathrm{x}$ & $\mathrm{x}$ & $\mathrm{x}$ & & \\
\hline Waist circumference & $\mathrm{x}$ & & & & & $\mathrm{x}$ & \\
\hline Blood pressure & $\mathrm{x}$ & & & $\mathrm{x}$ & & $\mathrm{x}$ & \\
\hline Fasting glucose & $\mathrm{x}$ & & & $\mathrm{x}$ & & $\mathrm{x}$ & \\
\hline Fasting lipids & $\mathrm{x}$ & & & $\mathrm{x}$ & & & $\mathrm{x}$ \\
\hline
\end{tabular}

*More frequent assessment may be warranted based on clinical status.

BMI, body mass index.

Copyright (c) 2004 American Diabetes Association. From Diabetes Care ${ }^{\circledR}$, Vol. 27, 2004; 596-601. Reprinted with permission from The American Diabetes Association.

morbidity and mortality is caused by CVD. ${ }^{2-4}$ As a result, the psychiatric literature is calling on psychiatrists to collaborate and coordinate with primary care physicians in screening for cardiometabolic risk factors and monitoring cardiometabolic medication side effects. ${ }^{5-7}$ Collaboration between mental and physical health care teams is not new but warrants emphatic renewal in light of these disturbing trends and the fact that some of this health deterioration is attributable to the negative cardiometabolic effects of prescribed treatment. An effective response to the health needs of those with SMI will require psychiatrists and primary care physicians to expanded their collaborative efforts and knowledge of each other's practices and treatments. The psychiatric care community has begun this work by discussing this topic in the literature, developing cardiometabolic screening guidelines (Table 1), and designing innovative, collaborative care models. Family physicians are particularly well suited to advance this important work and bring the discussion already under development in the field of psychiatry to the discipline of primary care.

\section{Understanding the Problem Excess Mortality}

An estimated 3\% of adults in the United States have SMI. This includes $1.5 \%$ with schizophrenia, $1 \%$ with bipolar disorder, and $1 \%$ with treatment refractory depression. ${ }^{8}$ Individuals with SMI have high rates of premature death, dying as much as 15 to 25 years younger than the general population, a trend that has accelerated in recent decades. ${ }^{4,9-12}$ Suicide remains an important cause of death among those with SMI, but most deaths result from natural causes, especially CVD, which occurs at a rate 2 to 3 times that of the general population. ${ }^{4,11,13,14}$

\section{Excess Morbidity}

Excess and increasing cardiovascular death is undoubtedly linked to significant and rising prevalence of cardiovascular risk factors. People with SMI suffer high rates diabetes, hypertension, obesity, and hyperlipidemia. ${ }^{15-18}$ The prevalence of CVD among people with schizophrenia or bipolar disorder is at least twice that of the general population. ${ }^{12,14,19,20}$ In one study of patients with schizophrenia, the prevalence of diabetes was estimated to be 4 times that of an age- and gendermatched control population, and the prevalence of hypertension was found to be approximately twice that of controls. ${ }^{15}$ In this same population, abdominal obesity affected $35 \%$ of men and $76 \%$ of women; among a comparison group, the prevalence was $25 \%$ and $57 \%$, respectively. ${ }^{21}$ People with schizophrenia also have less favorable cholesterol profiles. ${ }^{15}$ Estimates of the prevalence of metabolic syndrome among those with schizophrenia range from $22 \%$ to $60 \%, 1.8$ to 2.5 times that of those without mental illness. ${ }^{21}$ Studies comparing cardiometabolic disease among patients with schizophrenia and those with bipolar disorder show these conditions are equally prevalent in the 2 populations. $^{22-24}$

\section{Behavior, Life Style, and Age}

Although some excess morbidity associated with SMI is attributed to genetic predisposition and underlying psychopathology, a significant portion probably results from modifiable behaviors such as cigarette smoking, poor diet, and inactivity. ${ }^{2,14,25}$ People with SMI are at least twice as likely to smoke as those without mental illness, and as much as $75 \%$ to $90 \%$ of those with schizophrenia smoke. $^{26-28}$ Smokers with SMI tend to smoke 
Table 2. Second-Generation Antipsychotics and Metabolic Derangement ${ }^{5}$

\begin{tabular}{lccc}
\hline Drug & $\begin{array}{c}\text { Weight } \\
\text { Gain }\end{array}$ & $\begin{array}{c}\text { Risk for } \\
\text { Diabetes }\end{array}$ & $\begin{array}{c}\text { Worsening } \\
\text { Lipid Profile }\end{array}$ \\
\hline Clozapine & +++ & + & + \\
Olanzapine & +++ & + & + \\
Risperidone & ++ & $\mathrm{D}$ & $\mathrm{D}$ \\
Quetiapine & ++ & $\mathrm{D}$ & $\mathrm{D}$ \\
Aripiprazole* & \pm & - & - \\
Ziprasidone* $^{*}$ & \pm & - & - \\
\hline
\end{tabular}

*Newer drugs with limited data.

+ , increased effect (the more + symbols the greater the effect); - , no effect; D, discrepant results.

Copyright (C) 2004 American Diabetes Association. From Diabetes Care $^{\circledR}$, Vol. 27, 2004; 596-601. Reprinted with permission from The American Diabetes Association.

heavily and have more difficulty quitting. ${ }^{27,29}$ People with SMI are also more likely, compared with the general population, to eat poor diets and remain sedentary. ${ }^{26,30,31}$ Aging of the population with SMI adds to the growing rate of medical comorbidity seen in this population but cannot account for increasing mortality rates, which are age adjusted. ${ }^{17}$

\section{Medication and Care Access Imbalance Medication Side Effects}

A portion of the excess disease, and probably excess mortality, observed in populations with SMI results from the negative metabolic effects of newer "atypical" antipsychotic medications, now the dominant pharmacologic treatment for psychotic disorders. ${ }^{15,16,32}$ Atypical antipsychotics are associated with development of obesity, diabetes, and dyslipidemia. A meta-analysis of studies assessing the impact of antipsychotics on weight over just 10 weeks of treatment found mean gains of $4 \mathrm{~kg}$ with clozapine and olanzapine use and $2 \mathrm{~kg}$ with risperidone use (Table 2). ${ }^{33}$ The risk of impaired glucose metabolism and diabetes associated with atypical antipsychotics seems to be 1.2 to 5 times the risk of placebo or no treatment (Table 1). ${ }^{32}$ Clozapine and olanzapine increase serum triglycerides by $30 \%$ and $40 \%$, respectively, compared with pretreatment baseline and treatment with first generation antipsychotics. ${ }^{34}$ Reports of extreme triglyceride increases exist for both of these agents, but neither significantly impacts total cholesterol values (Table 1). ${ }^{34}$
The mechanisms by which atypical antipsychotics induce obesity and disrupt glucose and lipid metabolism are poorly understood; they likely occur through multiple pathways that include direct pharmacologic effects, compounded dietary indiscretion, and diminished activity. ${ }^{3-36}$ Much of the diabetes and hyperlipidemia seem related to treatment-associated obesity, but up to $25 \%$ of new cases occur independent of weight gain and obesity, suggesting some agents directly impact glucose and lipid metabolism. ${ }^{32,34,35}$

\section{Inadequate Access to and Use of Quality Health Care} Paradoxically superimposed on the iatrogenic disease that results from prescription medication access and use is a pattern of insufficient access to and use of quality health care. A recent study of people with schizophrenia revealed point prevalence of nontreatment was $30 \%$ for diabetes, $62 \%$ for hypertension, and $88 \%$ for hyperlipidemia. ${ }^{16}$ Mortality after myocardial infarction is relatively high in this population, and this higher mortality seems fully attributable to lower quality of care: less use of aspirin, beta-blockers, angiotensin-converting enzyme inhibitors, reperfusion, and smoking cessation counseling. ${ }^{37,38} \mathrm{~A}$ recent study found that people with schizophrenia experienced twice as many inpatient adverse events as those without this disease. ${ }^{39}$ Researchers at the Veterans Administration found mental illness negatively correlated with receipt of preventive health care and screening tests in that setting. ${ }^{40,41}$

\section{Obstacles to Care}

The poor quality of care observed in this population probably relates to patient characteristics, clinician behavior, and the system in which this care occurs. People with SMI are less likely than those without mental illness to seek care and adhere to prescribed treatments. ${ }^{2}$ Primary care clinicians may be uncomfortable or lack necessary skills to treat patients with SMI. ${ }^{42}$ Psychiatrists may not believe physical health is their responsibility or may not feel knowledgeable about physical medicine. ${ }^{43}$ Time constraints and competing demands perceived by physicians may limit willingness and ability to expand scope of practice. ${ }^{6,44,45}$ The seriously mentally ill may be less able than others to communicate symptoms, and physicians may question the veracity of complaints made by those with SMI. ${ }^{6}$ Within a busy primary care setting, patients 
with SMI and poor organizational skills may be effectively limited to few topics per visit. Visits may thus erroneously focus on acute symptoms rather than chronic illness, risks, and prevention. Therapeutic nihilism on the part of clinicians may limit interventional and educational efforts. Complex and fragmented care systems may be particularly difficult for those with SMI to effectively navigate. Fee-for-service reimbursement models that favor procedures, multiple short visits, and specialty care over longer encounters and primary care undoubtedly magnify these potential sources of limited care. $^{46}$

\section{Addressing the Problem}

Successes achieved through the chronic care model have taught us that health improvement will not occur through good clinical care alone. ${ }^{47}$ Eliminating these health disparities will require work on multiple levels including clinics, communities, public health agencies, insurers, and state and federal policy. For individual family physicians we propose, as an initial step, a unified effort at the clinical level through explicit recognition of the problem and interdisciplinary dialogue and collaboration. Creative models for such collaboration are being developed and tested. These efforts will be strengthened by further involvement of family physicians.

Family physicians are uniquely suited to address complex health issues for patients suffering with SMI. Our focus on family and community prepares us for this work and facilitates implementation of the chronic care model and experimentation with creative new care models. Our emphasis on holistic care and our efforts to maintain continuity make us a natural medical care anchor for patients with SMI. Our ability to provide procedures, preventive care, orthopedic treatment, gynecologic care, and acute and chronic disease management to all age groups helps minimize fragmentation of care for our patients, fragmentation that may be particularly prohibitive to people with SMI. Our expertise in care coordination, patient advocacy, and care planning may be exactly what is needed to begin reversing outcome disparities of this population.

\section{Integrated Care Models}

The literature on efforts to improve mental health care in the primary care setting is abundant, but evidence from efforts to improve physical health care of psychiatry patients is limited. Programs with this aim are heterogeneous and include: (1) physicians dually trained in psychiatry and physical medicine; ${ }^{48,49}(2)$ on-site medical consultation at a mental health care center; ${ }^{50}$ (3) collaborative care models that provide context and structure for regular communication between physical and mental health care teams; ${ }^{45,51,52}$ (4) nurse case managers who facilitate access to care, aid in decision making, and educate; $;^{7,53,54}$ and (5) facilitated referral models in which the psychiatric care team helps patients link to a primary care team through referral efforts. ${ }^{55,56}$ Each of these of these models aims in distinct ways to reduce barriers to physical care by connecting disconnected psychiatric and physical care services. The care model best suited to an individual practice will depend on practice location, size, affiliations, external support, funding/revenue sources, availability of community and academic psychiatrists, and prevalence of SMI within the population served (Table 3). Once engaged in this effort, family physicians' unique perspectives and practice experiences will probably give rise to new care models and improve those currently under development and study.

\section{Barriers to Integrated Care}

Although many agree that integrated care is a promising approach to the problem of excess morbidity and mortality among people with SMI, many obstacles hinder the development of such models. Financial barriers and the work required to create and sustain these programs may prove most challenging. Fee-for-service reimbursement schedules favor short office visits and thus discourage exploration of complex health conditions and risks. ${ }^{46}$ Behavioral health carve-outs, a popular financial model for separating budgets of physical and psychiatric care, probably limit efforts of integration by creating budgetary uncertainty or even budgetary conflict around the responsibilities of various clinical teams. ${ }^{45,53,56}$ Clinicians are not compensated directly for time spent communicating with colleagues. This creates a financial disincentive for complex care coordination. ${ }^{46}$ Establishing new collaboration and communication is involved work that requires commitment and time. Training programs may inadequately provide skills required for development and maintenance of such collaborative relationships. As a result, physicians may feel ill prepared to embark on such arrangements. 


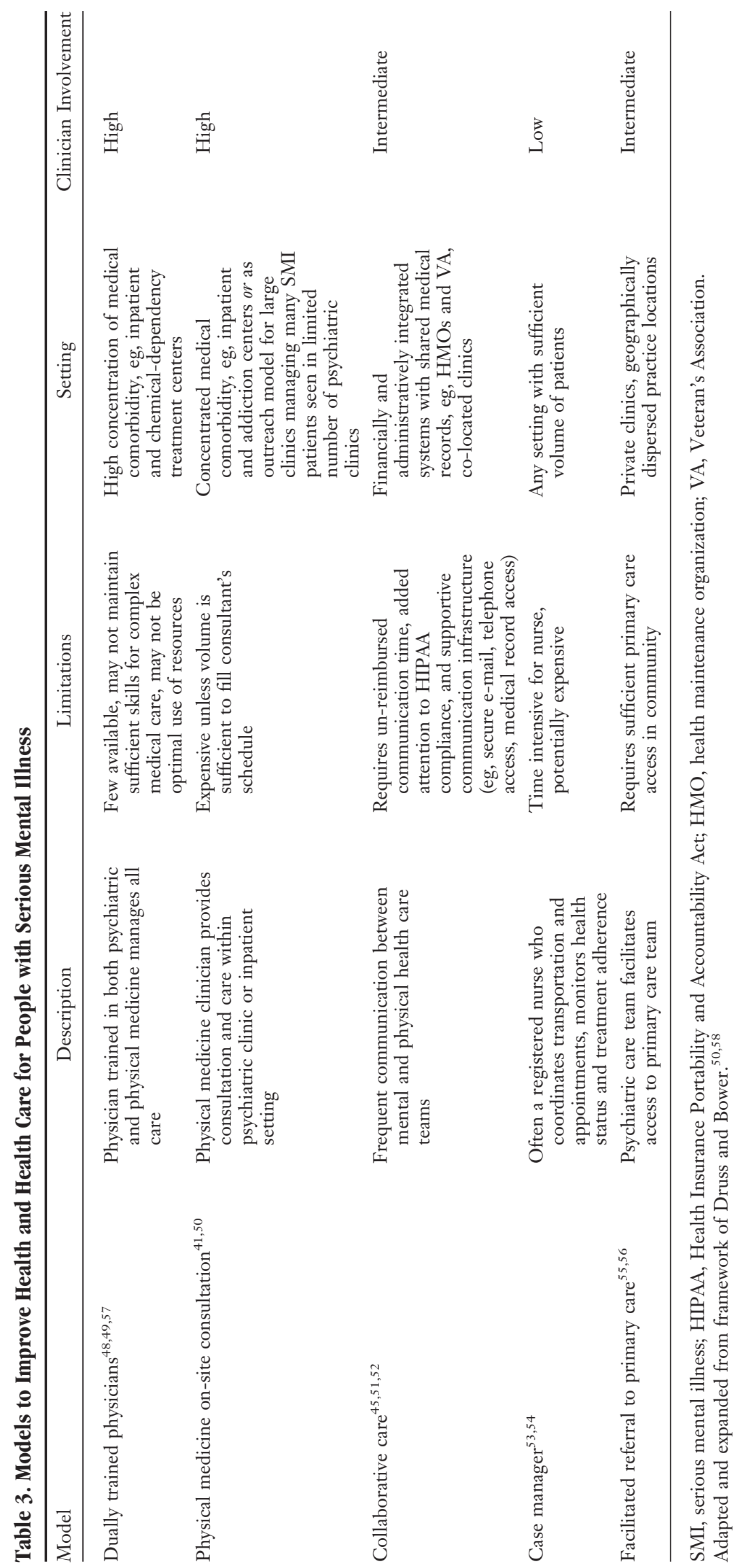




\section{What Individual Family Physicians Can Do}

Recently, Mary Seeman, ${ }^{59}$ a psychiatrist with 50 years' experience treating SMI, lamented the apparent trade off between autonomy conferred by deinstitutionalization and increasing premature mortality. Is this trade off inevitable? Might people with SMI have freedom and health? To this end, psychiatrists and primary care physicians can work collaboratively promoting interdisciplinary education, support, and models of care more effective for this population.

Potential strategies for family physicians committed to the health care of this population include:

1) Building integrated care models into practices through collaborative relationships with community and academic psychiatrists.

- Select practice-appropriate collaboration options (Table 3).

- Develop new models of care and collaboration.

- Identify a physician "champion" and communication point person for collaboration building.

- Select measurable outcomes to monitor effectiveness.

- Apply continuous quality improvement methods to the process. ${ }^{60}$

2) Treating medical comorbidities and high-risk behaviors aggressively, acknowledging that psychopathology will complicate but not prohibit such efforts.

- Establish SMI disease registries and monitoring protocols.

- Understand empiric evidence on behavior modification programs proven successful for people with SMI. ${ }^{61-72}$ Counsel and facilitate access to appropriate behavioral change programs for smoking cessation, weight loss and exercise.

- Track missed visits, practice outreach, optimize continuity of care, and facilitate treatment adherence.

- Consider open access to better match demand and needs of people with SMI.

3) Understanding psychopharmacologic treatment options, their side effects, and recommended monitoring guidelines (Tables 1 and 2). ${ }^{5}$
- Study the guidelines, review prescribing literature, and track updates.

- Know what others are prescribing and what the patient is taking.

- Set up automated monitoring reminder systems.

4) Attentively advocate for thorough, high-quality inpatient care.

- Advocate for appropriate diagnostic and therapeutic treatment.

- Address the risk of adverse events openly and advocate for enhanced attention to this risk.

5) Developing residency curricula on collaborative mental health care models.

- Develop such collaborations within residency clinics.

- Role model family medicine psychiatry collaboration.

- Include collaborative work in psychiatry rotations.

6) Reflect on personal tendencies toward therapeutic nihilism and find sources of support to bolster our commitment

- Use Bailant or other support groups for discussion.

- Share successes and study failures openly.

- Include the entire care team in this effort.

7) Advocating for reimbursement reform that recognizes the value of this complex work.

- Work through organizations such as the American Academy of Family Physicians to promote policy changes aimed at addressing the financial barriers to this complex work. Advocacy efforts should include distinct, reimbursed billing codes for care coordination and family meetings in the inpatient or outpatient setting.

8) Disseminating the design and results of collaborative efforts.

- Plan to study your work as a quality improvement effort or as clinical research.

- Share results through presentations and publications. 
Like all quality improvement efforts, the development of such models will be complex and iterative. The task may seem daunting, but the work is critically important, and family physicians are well suited to the task. To improve outcomes of those with SMI, the time is right for enhanced partnerships between family medicine and psychiatry. Family physicians already benefit from access to clinical support by psychiatrists. ${ }^{73}$ Psychiatrists acknowledge their responsibility to forge linkages to assure cardiometabolic monitoring and treatment for their patients with increasing risk of cardiometabolic disease and premature death. Thus both groups need the assistance of the other and have ample motivation to solidify collaboration for the benefit of this vulnerable population. Although this effort will not redress larger system failures and policy shortcomings, it is a starting point within reach of most practitioners and has potential to benefit those we are so overtly failing to serve with current care models.

The authors thank Dr. Lucy Candib for her review and advice on early drafts of this work.

\section{References}

1. National Heart Lung and Blood Institute. National Cholesterol Education Program Report. Implications of recent clinical trials for the National Cholesterol Education Program Adult Treatment Panel III Guidelines, 2004. Available at http://www. nhlbi.nih.gov/guidelines/cholesterol/atp3upd04.pdf. Accessed 22 January 2008.

2. Brown S, Inskip H, Barraclough B. Causes of the excess mortality of schizophrenia. Br J Psychiatry 2000;177:212-7.

3. Osby U, Correia N, Brandt L, Ekbom A, Sparen P. Time trends in schizophrenia mortality in Stockholm county, Sweden: cohort study. BMJ 2000;321: 483-4.

4. Saha S, Chant D, McGrath J. A systematic review of mortality in schizophrenia: is the differential mortality gap worsening over time? Arch Gen Psychiatry 2007;64:1123-31.

5. American Diabetes Association; American Psychiatric Association; American Association of Clinical Endocrinologists; North American Association for the Study of Obesity. Consensus development conference on antipsychotic drugs and obesity and diabetes. Diabetes Care 2004;27:596-601.

6. Goff DC. Integrating general health care in private community psychiatry practice. J Clin Psychiatry 2007;68(suppl 4):49-54.

7. Vreeland B. Bridging the gap between mental and physical health: a multidisciplinary approach. J Clin Psychiatry 2007;68(suppl 4):26-33.

8. Narrow WE, Regier DA, Norquist G, Rae DS, Kennedy C, Arons B. Mental health service use by Americans with severe mental illnesses. Soc Psychiatry Psychiatr Epidemiol 2000;35:147-55.

9. Brown S. Excess mortality of schizophrenia. A metaanalysis. Br J Psychiatry 1997;171:502-8.

10. Colton CW, Manderscheid RW. Congruencies in increased mortality rates, years of potential life lost, and causes of death among public mental health clients in eight states. Prev Chronic Dis 2006;3:A42.

11. Hansen V, Jacobsen BK, Arnesen E. Cause-specific mortality in psychiatric patients after deinstitutionalisation. Br J Psychiatry 2001;179:438-43.

12. Osby U, Correia N, Brandt L, Ekbom A, Sparen P. Mortality and causes of death in schizophrenia in Stockholm county, Sweden. Schizophr Res 2000;45: 21-8.

13. Hennekens CH, Hennekens AR, Hollar D, Casey DE. Schizophrenia and increased risks of cardiovascular disease. Am Heart J 2005;150:1115-21.

14. Osborn DP, Levy G, Nazareth I, Petersen I, Islam A, King MB. Relative risk of cardiovascular and cancer mortality in people with severe mental illness from the United Kingdom's General Practice Research Database. Arch Gen Psychiatry 2007;64:242-9.

15. Goff DC, Sullivan LM, McEvoy JP, et al. A comparison of ten-year cardiac risk estimates in schizophrenia patients from the CATIE study and matched controls. Schizophr Res 2005;80:45-53.

16. Nasrallah HA, Meyer JM, Goff DC, et al. Low rates of treatment for hypertension, dyslipidemia and diabetes in schizophrenia: data from the CATIE schizophrenia trial sample at baseline. Schizophr Res 2006;86:15-22.

17. Bartels SJ. Caring for the whole person: integrated health care for older adults with severe mental illness and medical comorbidity. J Am Geriatr Soc 2004; 52(12 Suppl):S249-57.

18. Sokal J, Messias E, Dickerson FB, et al. Comorbidity of medical illnesses among adults with serious mental illness who are receiving community psychiatric services. J Nerv Ment Dis 2004;192:421-7.

19. Osby U, Brandt L, Correia N, Ekbom A, Sparen P. Excess mortality in bipolar and unipolar disorder in Sweden. Arch Gen Psychiatry 2001;58:844-50.

20. Kilbourne AM, Brar JS, Drayer RA, Xu X, Post EP. Cardiovascular disease and metabolic risk factors in male patients with schizophrenia, schizoaffective disorder, and bipolar disorder. Psychosomatics 2007; 48:412-7.

21. McEvoy JP, Meyer JM, Goff DC, et al. Prevalence of the metabolic syndrome in patients with schizophrenia: baseline results from the Clinical Antipsychotic Trials of Intervention Effectiveness (CATIE) schizophrenia trial and comparison with national es- 
timates from NHANES III. Schizophr Res 2005;80: $19-32$.

22. Kilbourne AM, Cornelius JR, Han X, et al. Burden of general medical conditions among individuals with bipolar disorder. Bipolar Disord 2004;6:36873.

23. Birkenaes AB, Opjordsmoen S, Brunborg C, et al. The level of cardiovascular risk factors in bipolar disorder equals that of schizophrenia: a comparative study. J Clin Psychiatry 2007;68:917-23.

24. Laursen TM, Munk-Olsen T, Nordentoft M, Mortensen PB. Increased mortality among patients admitted with major psychiatric disorders: a registerbased study comparing mortality in unipolar depressive disorder, bipolar affective disorder, schizoaffective disorder, and schizophrenia. J Clin Psychiatry 2007;68:899-907.

25. Mortensen PB, Juel K. Mortality and causes of death in first admitted schizophrenic patients. Br J Psychiatry 1993;163:183-9.

26. Brown S, Birtwistle J, Roe L, Thompson C. The unhealthy lifestyle of people with schizophrenia. Psychol Med 1999;29:697-701.

27. Compton MT, Daumit GL, Druss BG. Cigarette smoking and overweight/obesity among individuals with serious mental illnesses: a preventive perspective. Harv Rev Psychiatry 2006;14:212-22.

28. Williams JM, Ziedonis D. Addressing tobacco among individuals with a mental illness or an addiction. Addict Behav 2004;29:1067-83.

29. Lasser K, Boyd JW, Woolhandler S, Himmelstein DU, McCormick D, Bor DH. Smoking and mental illness: a population-based prevalence study. JAMA 2000;284:2606-10.

30. Wildes JE, Marcus MD, Fagiolini A. Obesity in patients with bipolar disorder: a biopsychosocialbehavioral model. J Clin Psychiatry 2006;67:904-15.

31. McCreadie R, Macdonald E, Blacklock C, et al. Dietary intake of schizophrenic patients in Nithsdale, Scotland: case-control study. BMJ 1998;317: 784-5.

32. Newcomer JW. Second-generation (atypical) antipsychotics and metabolic effects: a comprehensive literature review. CNS Drugs 2005;19(suppl 1):1-93.

33. Allison DB, Mentore JL, Heo M, et al. Antipsychoticinduced weight gain: a comprehensive research synthesis. Am J Psychiatry 1999;156:1686-96.

34. Meyer JM, Koro CE. The effects of antipsychotic therapy on serum lipids: a comprehensive review. Schizophr Res 2004;70:1-17.

35. Henderson DC, Cagliero E, Copeland PM, et al. Glucose metabolism in patients with schizophrenia treated with atypical antipsychotic agents: a frequently sampled intravenous glucose tolerance test and minimal model analysis. Arch Gen Psychiatry 2005;62:19-28.

36. Newcomer JW. Abnormalities of glucose metabo- lism associated with atypical antipsychotic drugs. J Clin Psychiatry 2004;65(suppl 18):36-46.

37. Druss BG, Bradford WD, Rosenheck RA, Radford MJ, Krumholz HM. Quality of medical care and excess mortality in older patients with mental disorders. Arch Gen Psychiatry 2001;58:565-72.

38. Druss BG, Bradford DW, Rosenheck RA, Radford MJ, Krumholz HM. Mental disorders and use of cardiovascular procedures after myocardial infarction. JAMA 2000;283:506-11.

39. Daumit GL, Pronovost PJ, Anthony CB, Guallar E, Steinwachs DM, Ford DE. Adverse events during medical and surgical hospitalizations for persons with schizophrenia. Arch Gen Psychiatry 2006;63: 267-72.

40. Druss BG, Rosenheck RA, Desai MM, Perlin JB. Quality of preventive medical care for patients with mental disorders. Med Care 2002;40:129-36.

41. Druss BG, Rohrbaugh RM, Levinson CM, Rosenheck RA. Integrated medical care for patients with serious psychiatric illness: a randomized trial. Arch Gen Psychiatry 2001;58:861-8.

42. Lester H, Tritter JQ, Sorohan H. Patients' and health professionals' views on primary care for people with serious mental illness: focus group study. BMJ 2005;330:1122.

43. Newcomer JW, Nasrallah HA, Loebel AD. The Atypical Antipsychotic Therapy and Metabolic Issues National Survey: practice patterns and knowledge of psychiatrists. J Clin Psychopharmacol 2004; 24(5 suppl 1):S1-6.

44. Grumbach K, Bodenheimer T. A primary care home for Americans: putting the house in order. JAMA 2002;288:889-93.

45. Mechanic D. Approaches for coordinating primary and specialty care for persons with mental illness. Gen Hosp Psychiatry 1997;19:395-402.

46. Alfano E. Integration of Primary Care and Behavioral Health: Report on a Roundtable Discussion of Strategies fro Private Health Insurance. In: Carty, L (ed.). Washington, DC: Bazelon Center for Mental Health Law; 2005.

47. Bodenheimer T, Wagner EH, Grumbach K. Improving primary care for patients with chronic illness: the chronic care model, Part 2. JAMA 2002; 288:1909-14.

48. Druss BG. Improving medical care for persons with serious mental illness: challenges and solutions. J Clin Psychiatry 2007;68(suppl 4):40-4.

49. Golomb BA, Pyne JM, Wright B, Jaworski B, Lohr JB, Bozzette SA. The role of psychiatrists in primary care of patients with severe mental illness. Psychiatr Serv 2000;51:766-73.

50. Druss BG, von Esenwein SA. Improving general medical care for persons with mental and addictive disorders: systematic review. Gen Hosp Psychiatry 2006;28:145-53.

51. Katon W, Von Korff M, Lin E, et al. Collaborative 
management to achieve treatment guidelines. Impact on depression in primary care. JAMA 1995;273:102631.

52. Dietrich AJ, Oxman TE, Williams JW Jr, et al. Re-engineering systems for the treatment of depression in primary care: cluster randomised controlled trial. BMJ 2004;329:602.

53. Koyanagi C. Get it together: how to integrate physical and mental health care for persons with serious mental illness. Washington, DC: Bazelon Center for Mental Health Law; 2004.

54. Griswold KS, Servoss TJ, Leonard KE, et al. Connections to primary medical care after psychiatric crisis. J Am Board Fam Pract 2005;18:166-72.

55. Samet JH, Larson MJ, Horton NJ, Doyle K, Winter M, Saitz R. Linking alcohol- and drug-dependent adults to primary medical care: a randomized controlled trial of a multidisciplinary health intervention in a detoxification unit. Addiction 2003;98:509-16.

56. National Association of State Mental Health Program Directors Medical Directors Council. Integrating behavioral health and primary care services: opportunities and challenges for state mental health authorities. Alexandria (VA): National Association of State Mental Health Program Directors; 2005.

57. Doebbeling CC, Pitkin AK, Malis R, Yates WR. Combined internal medicine-psychiatry and family medicine-psychiatry training programs, 1999-2000: program directors' perspectives. Acad Med 2001;76: 1247-52.

58. Bower P, Gilbody S. Managing common mental health disorders in primary care: conceptual models and evidence base. BMJ 2005;330:839-42.

59. Seeman MV. An outcome measure in schizophrenia: mortality. Can J Psychiatry 2007;52:55-60.

60. Kritchevsky SB, Simmons BP. Continuous quality improvement. Concepts and applications for physician care. JAMA 1991;266:1817-23.

61. Addington J. Group treatment for smoking cessation among persons with schizophrenia. Psychiatr Serv 1998;49:925-8.

62. Addington J, el-Guebaly N. Group treatment for substance abuse in schizophrenia. Can J Psychiatry 1998;43:843-5.

63. Addington J, el-Guebaly N, Campbell $\mathrm{W}$, Hodgins DC, Addington D. Smoking cessation treatment for patients with schizophrenia. Am J Psychiatry 1998; 155:974-6.

64. Bartels SJ, Forester B, Mueser KT, et al. Enhanced skills training and health care management for older persons with severe mental illness. Community Ment Health J 2004;40:75-90.

65. Khazaal Y, Fresard E, Rabia S, et al. Cognitive behavioral therapy for weight gain associated with antipsychotic drugs. Schizophr Res 2007;91:169-77.

66. Chou KR, Chen R, Lee JF, Ku CH, Lu RB. The effectiveness of nicotine-patch therapy for smoking cessation in patients with schizophrenia. Int J Nurs Stud 2004;41:321-30.

67. Evans S, Newton R, Higgins S. Nutritional intervention to prevent weight gain in patients commenced on olanzapine: a randomized controlled trial. Aust N Z J Psychiatry 2005;39:479-86.

68. Hoffmann VP, Ahl J, Meyers A, et al. Wellness intervention for patients with serious and persistent mental illness. J Clin Psychiatry 2005;66:1576-9.

69. Littrell KH, Hilligoss NM, Kirshner CD, Petty RG, Johnson CG. The effects of an educational intervention on antipsychotic-induced weight gain. J Nurs Scholarsh 2003;35:237-41.

70. Mauri M, Castrogiovanni S, Simoncini M, et al. Effects of an educational intervention on weight gain in patients treated with antipsychotics. J Clin Psychopharmacol 2006;26:462-6.

71. Melamed Y, Stein-Reisner O, Gelkopf M, et al. Multi-modal weight control intervention for people with persistent mental disorders. Psychiatr Rehabil J 2008;31:194-200.

72. Wu RR, Zhao JP, Jin H, et al. Lifestyle intervention and metformin for treatment of antipsychotic-induced weight gain: a randomized controlled trial. JAMA 2008;299:185-93.

73. Levine S, Unutzer J, Yip JY, et al. Physicians' satisfaction with a collaborative disease management program for late-life depression in primary care. Gen Hosp Psychiatry 2005;27:383-91. 